\title{
КЛИНИЧЕСКИЕ ВАРИАНТЫ ГАСТРИНОМ; ТРУДНОСТИ ДИАГНОСТИКИ
}

\author{
Терещенко И.В.
}

ООО «Профессорская клиника», Пермь

ЦЕЛЬ: проанализировать клинические варианты гастрином, трудности диагностики.

МАТЕРИАЛЫ И МЕТОДЫ: определили уровень гастрина в крови иммуноферментным методом (норма 13-110 пг/мл) у 127 человек, обратившихся в поликлинику. При неоднократном обнаружении гипергастринемии >400 пг/мл исключали гастриному: проводили углубленное клиническое обследование и топическую диагностику опухоли.

РЕЗУЛЬТАТЫ: гастринома выявлена у 7 (5,5\%) обследованных. Классический синдром ЗоллингераЭллисона оказался в 3 случаях. Уровень гастрина в крови этих больных был 527-896 пг/мл. В течение 3-5 лет им выставляли диагноз и лечили без какого-либо эффекта по поводу язвенной болезни желудка и/или двенадцатиперстной кишки. H. Pylori при неоднократной проверке не был обнаружен. Еще в 3 случаях высокая гипергастринемия оказалась случайной находкой; у больных выявлена бессимптомная форма гастрином: не было болевого синдрома, диспепсических проявлений, пациенты считали себя здоровыми. При проведении ФГС обнаружен только поверхностный гастрит, хотя уровень гастрина в крови стабильно превышал 440-1100 пг/мл. Гастриномы у них выявлены в поджелудочной железе на границе тела и хвоста, имели четкую капсулу, округлую форму, диаметр опухолей не превышал 2,5 см. Учитывая, что гипергастринемия является мощным канцерогенным фактором, всем больным - и с синдромом Золлингер-Эллисона, и с бессимптомной формой - рекомендовано оперативное лечение. К настоящему времени с бессимптомной формой опухоли прооперированы две пациентки. Уровень гастрина у них пришел к норме, в том числе стал< 100 пг/мл у больной с гастринемией до хирургического лечения $>1100$ пг/мл. Особое внимание заслуживает пациентка с выявленной в терминальной стадии злокачественной гастриномой желудка. Впервые обратилась к врачу по поводу случайно выявленной гипергликемии. Диспепсических нарушений и болевого синдрома никогда не было. Несмотря на быстрое снижение массы тела за последние 5-6 месяцев, развитие кахексии, землистый цвет лица, анемию, терапевтом поставлен диагноз: «Сахарный диабет 2-го типа» и назначен метформин, который усугубил истощение. Проведена ФГС, выявлен рак тела желудка, кардии с переходом на нижнюю треть пищевода, прорастание в печень и панкреас. Опухоль исходила из G-клеток. Наступил летальный исход.

ВЫВОДЫ: помимо известных вариантов гастриномы - синдрома Золлингер-Эллисона и малосимптомных форм со стертой клинической симптоматикой - гастриномы могут протекать бессимптомно, проявляться только высокой гипергастринемией. Для своевременной диагностики гастрином главным является не эндоскопический контроль желудка и двенадцатиперстной кишки, а скрининг гастрина в крови. Пока невозможно объяснить, почему гипергастринемия не вызвала у больных с бессимптомной формой синдром Золлингер-Эллисона, однако, при этом сохраняется высокий риск канцерогенеза. Нельзя признать гастриному редкой патологией: при проверке гастринемии сплошным методом опухоль выявлена у 5,5\% обследованных.

КЛЮЧЕВЫЕ СЛОВА: нейроэндокринные опухоли желудочно-кишечного тракта, гастриномы, гастрин. 